\title{
Controllability of Kinematic Control Systems on Stratified Configuration Spaces
}

\author{
Bill Goodwine Joel Burdick \\ Division of Engineering and Applied Science \\ California Institute of Technology \\ Pasadena, California 91125
}

June 10, 1996

\section{Introduction}

Many interesting and important control systems evolve on stratified configuration spaces. Robotic systems, in particular, are of this nature. A legged robot has discontinuous equations of motion near points in the configuration space where each of its "feet" come into contact with the ground, and it is precisely the ability of the robot to lift its feet off of the ground that enables it to move about. Similarly, a robotic hand grasping an object often cannot reorient the object without lifting its fingers off of the object. Despite the obvious utility of such systems, however, a comprehensive means to analyze their controllability properties, to our knowledge, has not appeared in the literature.

For such systems, the equations of motion on each strata may change in a non-smooth, or even discontinuous manner, when the system moves from one strata to another. In such cases, traditional nonlinear controllability analyses are inapplicable because they rely upon differentiation in one form or another. Yet it is the discontinuous nature of such systems that is often their most important characteristic. Therefore, it is necessary to incorporate explicitly into a controllability analysis the non-smooth or discontinuous nature of these systems.

We present two controllability tests for stratified, kinematic control systems. We assume that the configuration manifold for the control system contains submanifolds with codimension one, in which the system is subjected to constraints in addition to those present outside the submanifold. Submanifolds of higher codimension may be defined by the intersection of two lower codimension submanifolds.

To use the first test, we consider the geometric relationship among involutive distributions associated with the various (sub)manifolds at a point in the configuration manifold. We note that the involutive distribution associated with a particular submanifold is more than simply the involutive closure of the control 
vector fields restricted to each submanifold. It is actually generated by a set of vector fields defined by a relationship between the vector fields on the submanifold and the vector fields on the manifold in which it is embedded. If there exists a nested sequence of submanifolds, each containing the point of interest, such that the sum of the associated involutive distributions evaluated at that point is the whole tangent space of the configuration manifold at that point, then the system is controllable.

We also present a controllability test using tools from exterior differential systems. The calculations in this test focus on the constraint equations, rather than the equations of motion. Because we have assumed that each submanifold has constraints in addition to those in the manifold in which it is embedded, fewer calculations are needed to use this test because many of the calculations will be repeated. Essentially the "dual" of the above test, controllability is determined by the geometric relationship among the derived flags associated with the same sequence of submanifolds described above.

\section{The Distribution Approach}

Throughout this paper we will be concerned with kinematic control systems of the form

$$
\dot{x}=g_{i}(x) u^{i}
$$

where $x \in M$, where $M$ is an $m$-dimensional manifold (the configuration manifold), $u=\left\{u^{1}, \ldots, u^{m}\right\} \in U \subset \mathbb{R}^{m}, U$ is the set of admissible controls and $g_{i}(x) \in \mathfrak{X}, \mathfrak{X}$ is the set of (not necessarily smooth, or even continuous) vector fields on $M$.

Assume that on a collection of submanifolds $S_{i} \subseteq M$, the system is subjected to constraints in addition to those present in $M$. For example, the legged robot will have additional constraints when one or more feet come into contact with the ground. Also, if $S_{i} \subseteq S_{j}$, assume that the system is subjected to constraints in $S_{i}$ in addition to those in $S_{j}$. Denote the set of constraints on $M$ by $\left\{\omega^{M, 1}, \ldots, \omega^{M, s}\right\}$. On a submanifold, $S_{i}$, denote the additional constraints with a superscript $S_{i}$. Thus, if $\operatorname{codim} S_{i}=1$, the set of constraints on $S_{i}$ is $\left\{\omega^{M, 1}, \ldots, \omega^{M, s}, \omega^{S_{i}, 1}, \ldots, \omega^{S_{i}, q}\right\}$. Also, assume that $S_{i}$ is locally described by the level set of a function $\Phi(x)=0$ and that the "constraint" $\mathbf{d} \Phi(x) \dot{x}=0$ is a constraint only on the control inputs. This means that there exists control inputs which ensure that the system evolves on the submanifold without leaving it.

We will write the equations of motion for the system at $x \in M, x \notin S_{i}$ as

$$
\dot{x}=g_{M, 1}(x) u^{M, 1}+\cdots g_{M, n}(x) u^{M, n},
$$

and the equations of motion for the system in one of the submanifolds at $x \in S_{i}$ as

$$
\dot{x}=g_{S_{i}, 1}(x) u^{S_{i}, 1}+\cdots g_{S_{i},(n-p)}(x) u^{S_{i},(n-p)},
$$


where $p$ depends upon the codimension of $S_{i}$ and the nature of the additional constraints imposed on the system in $S_{i}$. Note also that the form of the control vector fields $g_{i}$ are different because additional constraints are present on $S_{i}$.

On a submanifold of codimension 1, the system is subjected to additional constraints, and the equations of motion are given by an equation of the form of Equation 2. Since we have assumed that $\mathbf{d} \Phi$ is a constraint only on the control inputs, all the directions in which the system can flow in $S_{i}$ are given by the involutive closure of the distribution formed by the intersection of $\left.\bar{\Delta}_{M}\right|_{x}$ and $T_{x} S_{i}$ plus the control vector fields on $S_{j}$ of the form of Equation 2,

$$
\Delta_{S_{i}}=\operatorname{span}\left\{v: v=g_{S_{i}, j}, j=1, \ldots,(n-p) \text { or }\left.v \in \bar{\Delta}_{M}\right|_{x} \cap T_{x} S_{i}\right\},
$$

denoted by $\bar{\Delta}_{S_{i}}$. For a submanifold, $S_{j}$ with codimension greater than 1 , we similarly construct an involutive distribution by calculating the involutive closure of the distribution formed by the intersection of $\bar{\Delta}_{S_{i}}$ and $T_{x} S_{j}$ plus the intersection of $\bar{\Delta}_{S_{k}}$ and $T_{x} S_{j}$ plus the control vector fields given in the form of Equation 2,

$\Delta_{S_{j}}=\operatorname{span}\left\{v: v=g_{S_{j}}, j=1, \ldots(n-p-q),\left.v \in \bar{\Delta}_{S_{i}}\right|_{x} \cap T_{x} S_{j}\right.$, or $\left.\left.v \in \bar{\Delta}_{S_{k}}\right|_{x} \cap T_{x} S_{j}\right\}$

where $S_{i}$ and $S_{k}$ are the two manifolds whose intersection defines $S_{j}$. We similarly denote the involutive closure of this distribution by $\bar{\Delta}_{S_{j}}$.

Now we can state our first controllability result.

PROPOSITION 2.1 If there exists a nested sequence of submanifolds

$$
S_{p_{i}} \subseteq S_{(p-1)_{i}} \subseteq \cdots \subseteq S_{1_{i}} \subseteq M
$$

such that the associated involutive distributions satisfy

$$
\left.\bar{\Delta}_{M}\right|_{x}+\left.\sum_{j=1}^{p} \bar{\Delta}_{S_{j_{i}}}\right|_{x}=T_{x} M
$$

then the system is STLC.

REMARK 2.2 In the nested sequence of manifold, the first subscript is the codimension of the submanifold. This subscript is also subscripted because there may be multiple submanifolds with the same codimension at the point $x$. If there are multiple submanifolds with the same codimension, this sequence only contains one of them.

REMARK 2.3 The idea behind the proof is simple. Figure 1 pictorially illustrates $M$, and one submanifold, $S_{i}$, and the associated involutive closures of the distributions associated with each regime. Note that the symbols for the involutive distributions are pointing to the manifolds to which they are the tangent space. If the system starts at a point $x_{0} \in S_{i}$, then the set of points it can reach in $S_{i}$ is represented by the lines (submanifolds) in $S_{i}$. If these submanifolds transversely intersect the manifolds which comprises the foliation associated with $\bar{\Delta}_{M}$, then these lines provide a means to locally flow to any leaf in the foliation defined by $\bar{\Delta}_{M}$. 


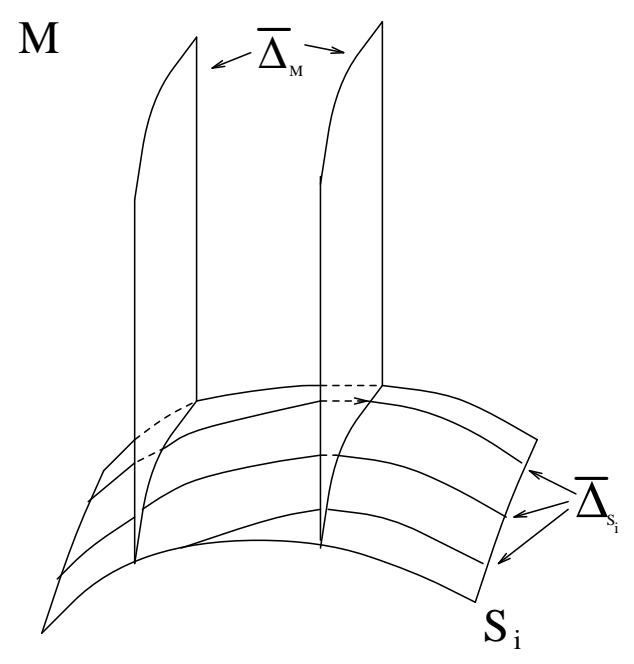

Figure 1. Controllability of a Stratified System

REMARK 2.4 Often in applications the submanifolds upon which the system is subjected to additional constraints will often be a physical boundaries, which the system cannot penetrate. In such a case, we have to redefine a neighborhood of a point $x_{0}$ contained in the boundary to be the union of the portion of the standard neighborhood on the "allowable" side of the manifold with the intersection of the standard neighborhood with the boundary. Proposition 2.1 still applies, however, due to the fact that we have assumed that we can directly control whether or not the system will flow onto or off of the manifold.

\section{The Exterior Differential Systems Approach}

Let $I=\operatorname{span}\left\{\omega^{1}, \ldots, \omega^{n}\right\}$ be a smooth codistribution on $M$, where the $\omega$ 's are the one forms describing the constraints. The derived flag is a construction which describes the integrability properties of the ideal generated by $I$. It follows from Frobenius' Theorem that $I^{(N)}$ is the largest integrable subsystem contained in $I$, therefore, if $I^{(N)}$ is not empty, there exists functions $h_{1}, \ldots, h_{r}$ such that $\left\{\mathbf{d} h_{i}\right\} \subset\{I\}$. Thus, if the bottom derived flag is not empty, there exists functions which describe a foliation of the state space.

Recall that on $M$, we have the collection of constraints $\left\{\omega^{M, 1}, \ldots, \omega^{M, s}\right\}$, and on a codimension 1 submanifold $S_{i}$ we have the collection of constraints $\left\{\omega^{M, 1}, \ldots, \omega^{M, s}, \omega^{S_{i}, 1}, \ldots, \omega^{S_{i}, q}\right\}$, which is equivalent to the set of constraints $\left\{\omega^{M, 1}, \ldots, \omega^{M, s}, \mathbf{d} \Phi, \omega^{S_{i}, 1}, \ldots, \omega^{S_{i}, q}\right\}$ on $M$. Let $I_{M}^{\left(N_{M}\right)}$ be the bottom derived flag for the constraints on $M$. Recall that on a submanifold, the system can momentarily move off of the submanifold to move in a direction contained in 
$\bar{\Delta}_{M} \cap T_{x} S_{i}$. In this case, the set of constraints to which the system is subjected is $\left\{\mathbf{d} \Phi, I_{M}^{\left(N_{M}\right)} \cap\left\{\omega^{M, 1}, \ldots, \omega^{M, s}, \mathbf{d} \Phi, \omega^{S_{i}, 1}, \ldots, \omega^{S_{i}, q}\right\}\right.$. Let $I_{S_{i}}^{\left(N_{S_{i}}\right)}$ be the bottom derived flag for this set of constraints. Similarly to the manner of construction in the previous section, we can calculate derived flags associated with each submanifold $S_{i}$.

Proposition 3.1 If there exists a nested sequence of submanifolds

$$
S_{p_{i}} \subseteq S_{(p-1)_{i}} \subseteq \cdots \subseteq S_{1_{i}} \subseteq M
$$

such that the associated derived flags satisfy

$$
\operatorname{span}\left(I_{M}^{\left(N_{M}\right)}\right) \bigcap \operatorname{span}\left(I_{S_{1_{i}}}^{\left(N_{S_{1}}\right)}\right) \bigcap \cdots \bigcap \operatorname{span}\left(I_{S_{p_{i}}}^{\left(N_{S_{p_{i}}}\right)}\right)=0,
$$

then the system is $S T L C$.

\section{An Example}

We illustrate the application of the first test by presenting the following example.

The following example is adapted from Kelly and Murray [7]. Consider the six-legged robot shown in Figure 2. Assume that the robot walks with a tripod gait, alternating movements of legs $1-4-5$ with movements of legs 2-3-6. Suppose that

$$
\begin{gathered}
\dot{x}=\cos \theta\left(\alpha\left(h_{1}\right) u_{1}+\beta\left(h_{2}\right) u_{2}\right) \\
\dot{y}=\sin \theta\left(\alpha\left(h_{1}\right) u_{1}+\beta\left(h_{2}\right) u_{2}\right) \\
\dot{\theta}=l \alpha\left(h_{1}\right) u_{1}-l \beta\left(h_{2}\right) u_{2} \\
\dot{\phi}_{1}=u_{1} \\
\dot{\phi}_{2}=u_{2} \\
\dot{h}_{1}=v_{1} \\
\dot{h}_{2}=v_{2}
\end{gathered}
$$

where $(x, y, \theta)$ represents the planar position of the center of mass, $\phi_{i}$ is the angle of the legs and $h_{i}$ is the height of the legs off the ground.

The functions $\alpha\left(h_{1}\right)$ and $\beta\left(h_{2}\right)$ are defined by

$$
\alpha\left(h_{1}\right)= \begin{cases}1 & \text { if } h_{1}=0 \\ 0 & \text { if } h_{1}>0\end{cases}
$$

The function $\beta\left(h_{2}\right)$ is the same, but as a function of $h_{2}$.

Now if all legs are in contact with the ground, the equations of motion are

$$
\left(\begin{array}{c}
\dot{x} \\
\dot{y} \\
\dot{\theta} \\
\dot{\phi}_{1} \\
\dot{\phi}_{2}
\end{array}\right)=\left(\begin{array}{cccc}
\cos \theta & \cos \theta & 0 & 0 \\
\sin \theta & \sin \theta & 0 & 0 \\
l & -l & 0 & 0 \\
1 & 0 & 0 & 0 \\
0 & 1 & 0 & 0
\end{array}\right)\left(\begin{array}{l}
u_{1} \\
u_{2} \\
u_{3} \\
u_{4}
\end{array}\right)
$$




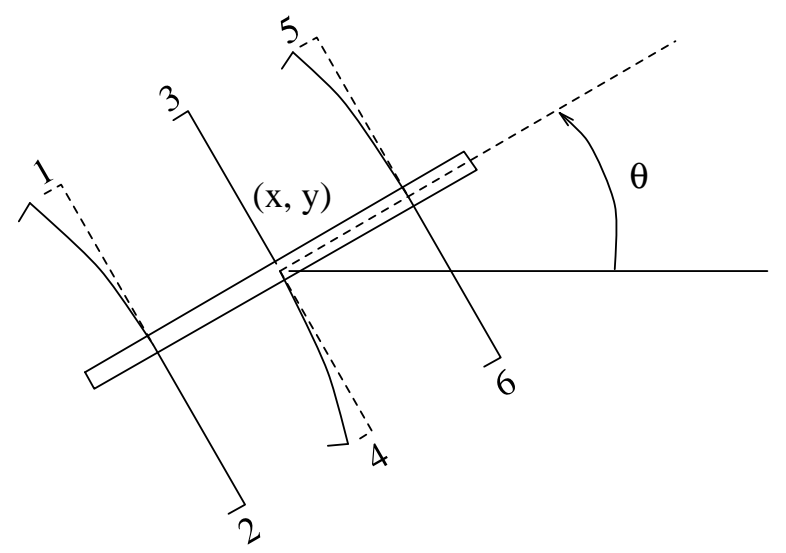

Figure 2. Six Legged Robot

where $u_{3}$ and $u_{4}$ are constrained to be 0 . Note that if we let $f$ represent the first column, and $g$ the second column, then

$$
[f, g]=\left(\begin{array}{c}
-2 l \sin \theta \\
2 l \cos \theta \\
0 \\
0 \\
0
\end{array}\right)
$$

Clearly, with all the legs in contact with the ground, we have generated enough directions to span the $(x, y, \theta)$ directions, but not without control over all the leg heights and angles.

If leg 1 is in contact with the ground, but leg 2 is not in contact, the equations of motion are

$$
\left(\begin{array}{c}
\dot{x} \\
\dot{y} \\
\dot{\theta} \\
\dot{\phi}_{1} \\
\dot{\phi}_{2} \\
\dot{h}_{2}
\end{array}\right)=\left(\begin{array}{cccc}
\cos \theta & 0 & 0 & 0 \\
\sin \theta & 0 & 0 & 0 \\
l & 0 & 0 & 0 \\
1 & 0 & 0 & 0 \\
0 & 1 & 0 & 0 \\
0 & 0 & 0 & 1
\end{array}\right)\left(\begin{array}{l}
u_{1} \\
u_{2} \\
u_{3} \\
u_{4}
\end{array}\right)
$$

where $u_{3}$ is constrained to be 0 .

Finally, if leg 2 is in contact with the ground and leg 1 is not, then the 
equations of motion are

$$
\left(\begin{array}{c}
\dot{x} \\
\dot{y} \\
\dot{\theta} \\
\dot{\phi}_{1} \\
\dot{\phi}_{2} \\
\dot{h}_{1}
\end{array}\right)=\left(\begin{array}{cccc}
0 & \cos \theta & 0 & 0 \\
0 & \sin \theta & 0 & 0 \\
0 & -l & 0 & 0 \\
1 & 0 & 0 & 0 \\
0 & 1 & 0 & 0 \\
0 & 0 & 1 & 0
\end{array}\right)\left(\begin{array}{l}
u_{1} \\
u_{2} \\
u_{3} \\
u_{4}
\end{array}\right)
$$

where $u_{4}$ is constrained to be 0 .

If none of the legs are in contact with the ground, we have

$$
\left(\begin{array}{c}
\dot{x} \\
\dot{y} \\
\dot{\theta} \\
\dot{\phi}_{1} \\
\dot{\phi}_{2} \\
\dot{h}_{1} \\
\dot{h}_{2}
\end{array}\right)=\left(\begin{array}{llll}
0 & 0 & 0 & 0 \\
0 & 0 & 0 & 0 \\
0 & 0 & 0 & 0 \\
1 & 0 & 0 & 0 \\
0 & 1 & 0 & 0 \\
0 & 0 & 1 & 0 \\
0 & 0 & 0 & 1
\end{array}\right)\left(\begin{array}{l}
u_{1} \\
u_{2} \\
u_{3} \\
u_{4}
\end{array}\right) .
$$

Now, if we take all the columns from Equation 7, the first two columns from Equation 3 and Equation 4, and combine them to form a distribution, it spans $T_{x} M \quad \forall x \in M$, which clearly satisfies the hypotheses of Proposition 2.1. Therefore, the system is STLC.

\section{References}

[1] R. Abraham, J. E. Marsden, and T. Ratiu. Manifolds, Tensor Analysis, and Applications. Springer-Verlag, second edition, 1988.

[2] Ralph Abraham and Jerrold E. Marsden. Foundations of Mechanics. Addison Wesley, second edition, 1978.

[3] William M. Boothby. An Introduction to Differentiable Manifolds and Reimannian Geometry. Academic Press, second edition, 1986.

[4] R. W. Brockett. Smooth multimode control systems. - , pages $103-110$.

[5] Harley Flanders. Differential Forms with Applications to the Physical Sciences. Dover, 1989.

[6] Alberto Isidori. Nonlinear Control Systems. Springer-Verlag, second edition, 1989.

[7] Scott D. Kelly and Richard M. Murray. Geometric phases and robotic locomotion. J. Robotic Systems, 1994. To appear.

[8] H. Nijmeijer and A. J. van der Schaft. Nonlinear Dynamical Control Systems. Springer-Verlag, 1990. 
[9] E.D. Sontag. Mathematical Control Theory. Springer-Verlag, 1990. 